\title{
Survival and Morphology of Transplanted Astrocytes in Normal and Brain-Damaged Rats
}

\author{
Z. Fulop, L. Lescaudron, J. Chachaj, R.L. Sutton, H.M. Geller* and D.G. Stein \\ Brain Research Laboratory, Rutgers University, Newark, NJ 07102 and \\ *UMDNJ-Robert Wood Johnson Medical School, Piscataway, NJ 08854, USA
}

Neurotoxic lesions of nucleus basalis magnocellularis (NBM) in rats cause depletion of acetylcholine $(\mathrm{ACh})$ in neocortex $(\mathrm{Ncx})$ and deficits in passive avoidance (PA) behavior. Such deficits in behavior can be used to evaluate treatments designed to promote functional recovery. Because cultured astrocytes produce, store and release trophic factors known to enhance the rescue of damaged neurons, they were selected for grafting into the damaged NBM. Astrocytes were implanted either directly into the damaged NBM or into the cholinergic cortical projection areas of the NBM. This study evaluates the role of cultured rat primary astrocytes in protecting structures of the central nervous system (CNS) affected by neurotoxic damage of the NBM.

Adult male albino rats [Sprague-Dawley (CD), Charles River Labs.] were used in this study. Under sodium pentobarbital $(50 \mathrm{mg} / \mathrm{kg}$ b.w.) anesthesia, a unilateral injection of ibotenic acid $(0.5 \mu \mathrm{l} ; 15 \mu \mathrm{g} / \mu \mathrm{l})$ was made into the left NBM over a 4 minute duration. Type-1, purified cultured rat primary astrocytes obtained from newborn, $C D$ rat cortex, were prelabeled with fluorescent dye bis-benzimide and transplanted into adult recipient brains. Transplantation of astrocytes into NBM or cortex of NBM damaged animals followed $30 \mathrm{~min}$ after injection of ibotenic acid. Location of the transplants and the experimental conditions of the host animals were as follows:

1. Transplantation into NBM of intact animals $(n=4)$;

2. Transplantation into Ncx of intact animals $(\mathrm{n}=5)$;

3. Transplantation into NBM of NBM damaged animals $(n=22)$;
4. Transplantation into ipsilateral Ncx of NBM damaged animals $(n=4)$.

Animals were killed by transcardial perfusion at days $14(n=42)$ and $60(n=9)$ after surgery. Frozen, serial sections were cut at $30 \mu \mathrm{m}$ thickness. Sections were processed for thionin, acetylcholine-esterase (AChE), cytochrome oxidase (CYO) and for glial fibrillary acidic protein (GFAP) immunohistochemistry using the PAP method.

Injection of ibotenic acid into NBM resulted in activation of the astrocytes detectable by GFAP immunoreaction after 14 days survival in areas including ventral pallidum, substantia innominata, part of the caudate/putamen, bed nucleus of stria terminalis and the reticular thalamic nucleus. The lesion also resulted in a dramatic reduction of the number of neurons in the injection area and $\mathrm{ACh}$ depletion in the ipsilateral neocortex as seen by reduction of $\mathrm{AChE}$ reactivity. At 14 days postgrafting into NBM or cortex, the transplanted astrocytes could be identified by their intensive fluorescence. Astrocytes transplanted into intact NBM or cortex survived in all cases. Astrocytes injected into the NBM area previously infused with ibotenic acid did not survive; only diffuse fluorescing masses of dead cells were seen in the lesion cavity.

Although surviving astrocytes were found primarily in the cavities formed at the tip of the injection needle, a number of them also migrated into host tissue, but remained in close vicinity to the transplantation cavity. Astrocytes transplanted into the neocortex of animals with NBM lesions produced an extended area of cortical necrosis, causing either large, empty cavities 
or loose, areolar tissue-masses filled with intensively fluorescent cells. Staining by GFAPimmunoreaction of bis-benzimide fluorescing tissue sections showed no double-labeled cells. Bis-benzimide-positive cells remained roundshaped with no detectable GFAP-positive processes. By 60 days postgrafting, most of the detectable fluorescent cells had disappeared. The transplantation cavities were filled at this time with extensive, GFAP-positive, glial scar tissue. Some of the round-shaped bis-benzimide positive cells appeared to be too large to be characterized as the formerly transplanted astrocytes; instead they showed features of monocyte/macrophage cell type.

On the basis of these results we may conclude that: 1) Implants of bis-benzimide prelabeled, purified, primary astrocytes can survive at least 14 days in the host brain but show low migration activity during this period; 2) Bis-benzimide prelabeled, implanted astrocytes cannot be detected 60 days after transplantation; 3 ) This fact may be due to degradation of the dye or to the death of the implanted cells. Bis-benzimide may also be toxic to these cells and interfere with their normal metabolic function, giving a distorted impression of their effects on recovery in brain damaged subjects; 4) Ibotenic acid at 15 $\mu \mathrm{g} / \mu \mathrm{l}$ concentration may be lethal for astrocytes when they are grafted into the vicinity of this substance; 5) The acetylcholine-depleted neocortex is severely damaged by astrocytes implanted into the projection area of the damaged NBM. Therefore it is unlikely that this type of grafting would produce therapeutic effects in the treatment of neurotoxic injury of NBM.

Supported by GENRE Corporation. 

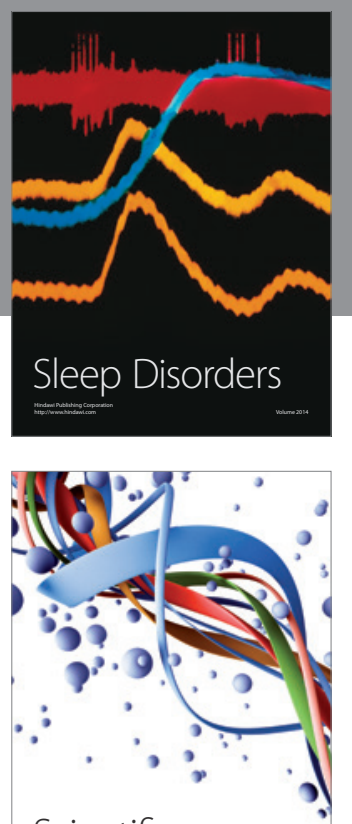

Scientifica
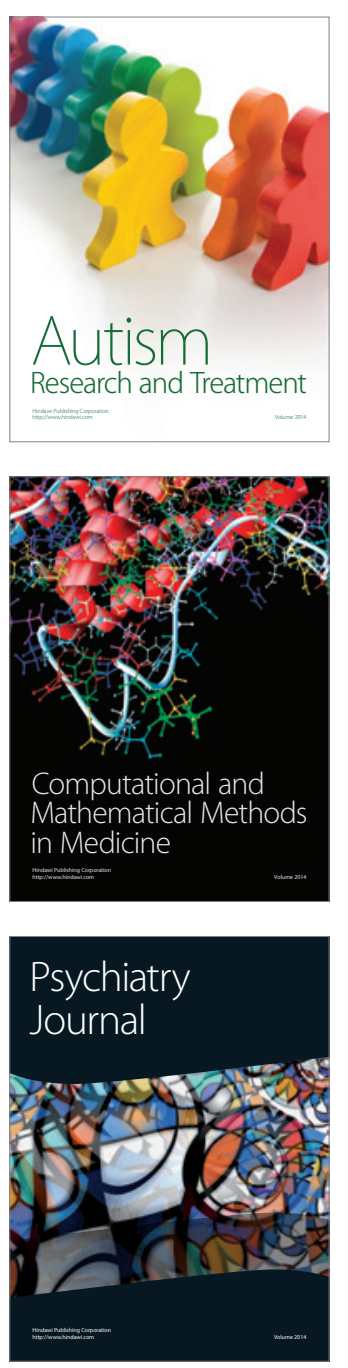
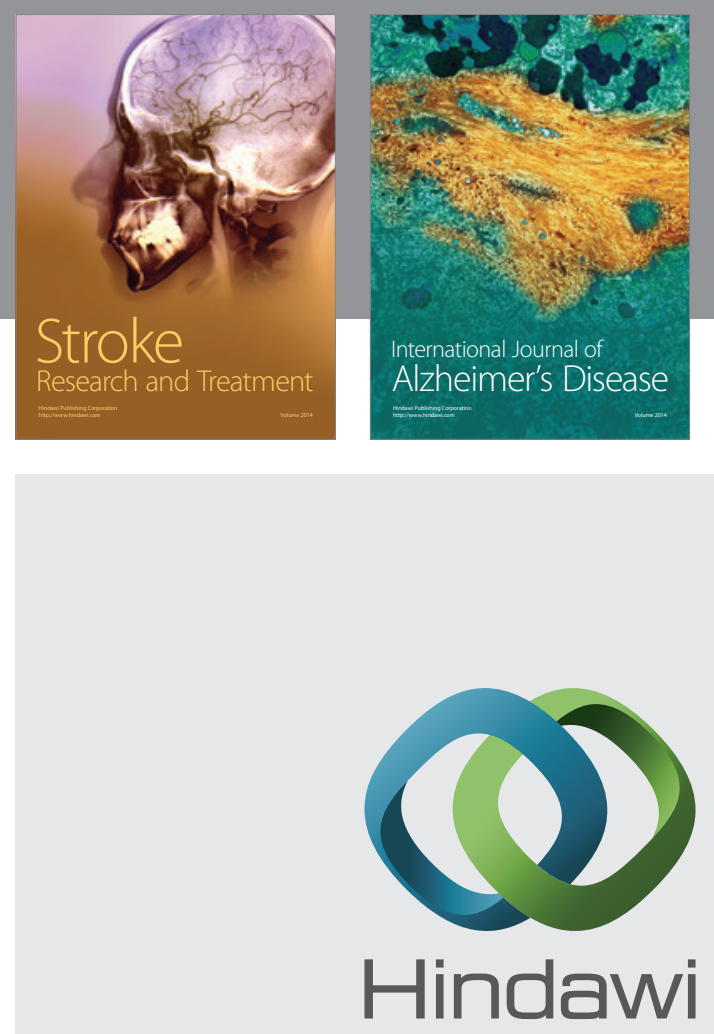

Submit your manuscripts at

http://www.hindawi.com
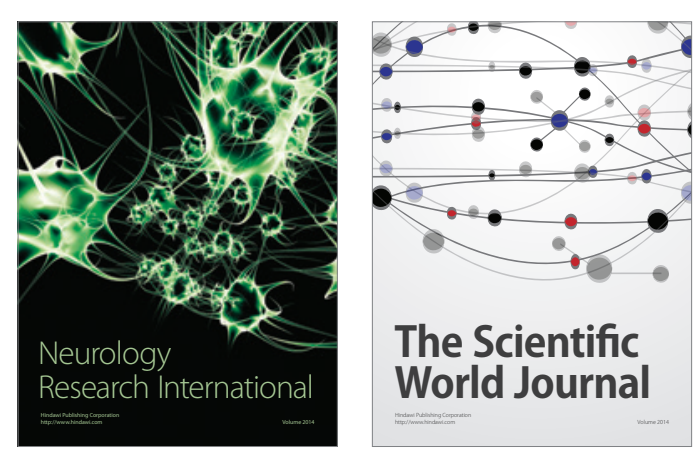

The Scientific World Journal

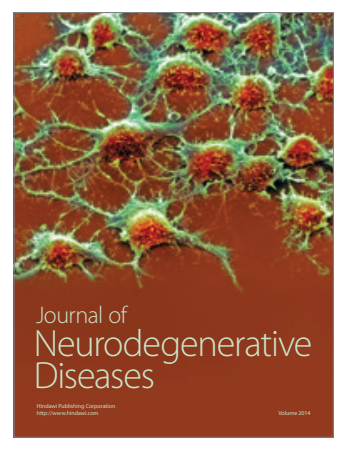

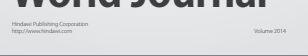

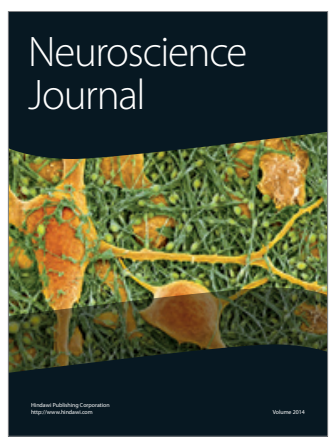

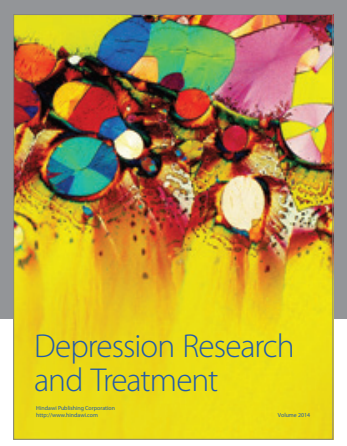
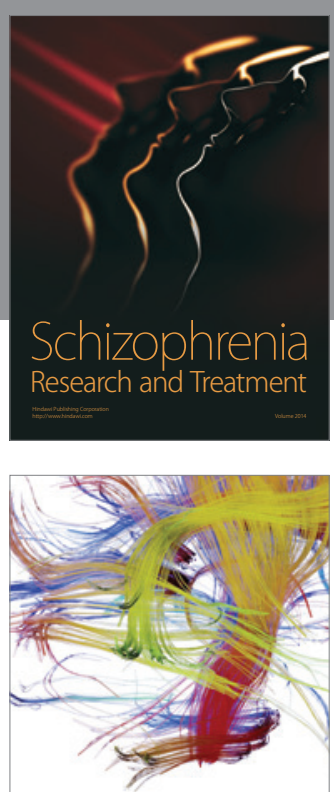

Brain Science

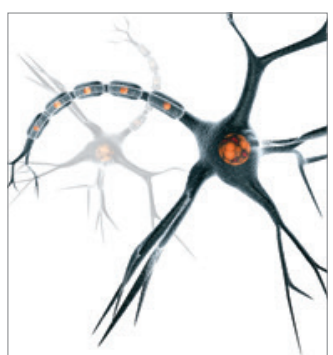

Neural Plasticity
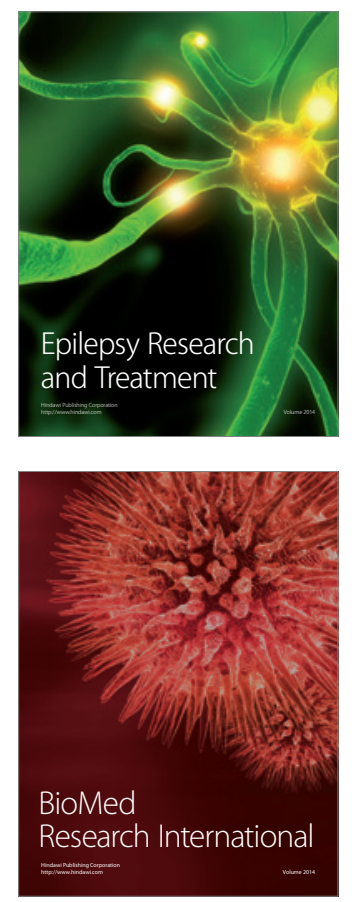

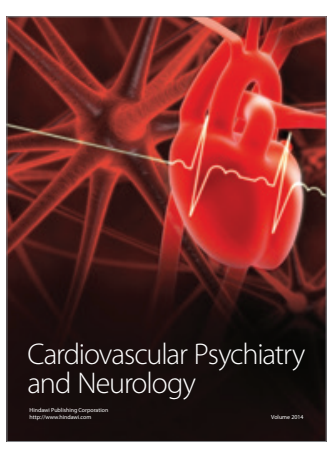

Parkinson's

Disease
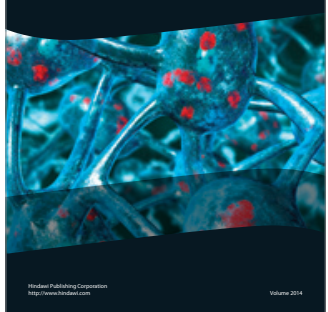